the same service in respect to calcium when it is diminished. We have encountered numerous instances in which a low percentage of about $7 \mathrm{mg}$. has been increased to more than $10 \mathrm{mg}$. by irradiations for from two to three. weeks with the carbon arc light. Only an extensive study can determine whether this reaction indicates that there is likewise a seasonal tide in infants of the calcium of the blood. In general, our figures for calcium are somewhat lower for the early spring than for June. The greater stability of calcium, however, as compared to the inorganic phosphate of the blood, would lead us to expect far less fluctuation.

As is well known, one of the disorders that manifest a striking seasonal incidence is tetany. The peak of its incidence occurs somewhat later in the spring than that of rickets. It is associated with a diminished content of calcium in the blood, which is especially low in its relation to the inorganic phosphate. In many cases there is an inverse ratio between the calcium and the phosphate content of the blood. This relationship may be illustrated by the case of an exceptionally well nourished infant, aged 11 months, who, March 8, had a content of $12.5 \mathrm{mg}$. per cent. of calcium and $3.54 \mathrm{mg}$. per cent. of inorganic phosphate in the blood. May 22, these amounts had changed to $7.55 \mathrm{mg}$. for calcium and $4 \mathrm{mg}$. for phosphate; the calcium had fallen markedly, whereas the phosphate had risen. Carbon arc irradiation was then given, and by June 8 the calcium had risen to $10 \mathrm{mg}$. and the phosphate to 4.58 mg. per cent. In other words, with the marked fall of the calcium in May there was a concomitant rise of phosphate. Such occurrences, which are not exceptional, suggest that the seasonal increase of phosphate, beginning in April and ascending still higher in May, tends in some cases to the development of tetany late in the spring.

This seasonal variation evidently indicates that the chemical constitution of our blood is not constant for all periods of the year; this probably holds true for other tissues. Marked fluctuations of this character may be discovered only in young children, in whom the body has to make provision not only for the metabolism associated with maintenance, but also for that demanded by rapid growth, a mechanism which necessarily involves a tendency to wider deviations from the normal. It is quite possible that "the phosphate blood tide" is only one of many seasonal variations that take place in the body. This aspect of metabolism has not been the subject of investigation, but it would seem to be a promising field, and a systematic study of the blood from various points of view-chemical, immunologic, etc.-might well be undertaken on animals and on man, and may reveal unknown seasonal peculiarities. In this connection, a variation in the iodin content of the thyroid gland of animals may be referred to. Some years ago, Seidell and Fenger ${ }^{7}$ showed that the average iodin content of the healthy thyroid gland of sheep, of cattle and of hogs is, in general, about three times as large for the months from June to November, as for the months from December to May. Another investigation which borders on this subject is that of Reid Hunt, ${ }^{8}$ who found that guinea-pigs were decidedly more resistant to acetonitril poisoning in the spring and summer than in the winter, and who concluded

7. Seidell, A., and Fenger, F.: Seasonal Variation in the Iodine Content of the Thyroid Gland, J. Biol. Chem. 13:517, 1913.

8. Hunt, Reid: The Effects of a Restricted Diet and of Various Diets upon the Resistance of Animals to Certain Poisons, Bull. 69, Hyg. Lab., U. S. P. H. S., 1910 . that this difference probably was associated with some seasonal variation in metabolism.

Undoubtedly climatic conditions exert a pronounced influence on general nutrition. For many years, clinicians have insisted on the obscure but definite relationship between seasonal changes and certain pathologic disorders, although scientific medicine could not substantiate their empiric observations. A striking example of the fundamental influence of the seasons on normal nutrition, and one which is subject to mathematical verification, is the seasonal periodicity of growth in length which is manifested in childhood. The investigations of Malling-Hansen and of others have shown that children grow far more rapidly in the spring and summer than during the winter months. Such seasonal activity is all the more significant when we bear in mind the fundamental character of the impulse required to bring about growth in length.

It is quite possible that chemical alterations of the tissues, induced by seasonal meteorological variations, may exert an influence not only on nutrition, but also on infection. For example, the low content of inorganic phosphate that occurs in the early spring and is closely associated with rickets may play a rôle in the susceptibility to infection which accompanies this disorder. Furthermore, seasonal alterations of the blood many bear a relationship to the vagaries in incidence of the epidemic diseases, and should be considered in connection with epidemiologic studies on animals and on man.

16 West Eighty-Sixth Street.

\section{MANAGEMENT OF HEAD INJURIES WITH REAL OR POTENTIAL BRAIN DAMAGE}

\section{WITH SPECIAL REFERENCE TO THE VALUE OF SATURATED SOLUTIONS OF MAGNESIUM SULPHATE AND SODIUM CHLORID}

CHARLES E. DOWMAN, M.D.

Assistant Professor of Surgery, in Charge of Neurologic Surgery, Emory University School of Medicine

ATLANTA, Ga.

During the last year, the relatively large number of cases of head injury admitted to the Grady Memorial Hospital (colored division) has afforded an unusual opportunity to work out a definite policy in the management of such cases, and one attended with a degree of success justifying this review.

\section{CLASSIFICATION OF CASES} thus :

We have learned to classify cases of head injury

A. Massive brain injury, with evidence of rapid exhaustion of the medullary centers, and death within one to several hours after admission.

B. Definite evidence of middle meningeal hemorrhage.

C. Simple or compound depressed fracture, with localized brain contusion, with or without indriven bone fragments.

D. Classic manifestations of rapidly increasing intracranial pressure which are well within the period of medullary compensation.

E. Definite evidence of brain injury exhibiting no classic findings of acutely increasing intracranial pressure, yet of the type that experience has shown is liable to develop gradually increased intracranial pressure due to fluid accumulation. 
F. So-called "concussion" with no evidence of gross brain damage.

G. Depressed fracture of a mild degree, giving rise to no symptoms whatsoever.

H. Scalp lacerations, without damage to the underlying structures.

\section{TREATMENT OF DIFFERENT CASE GROUPS}

Before determining the type of treatment, we attempt, as far as possible, to classify the case. In many cases, the eventual decision may be delayed until the patient has been observed for several hours.

A classification under $\mathrm{A}, \mathrm{B}, \mathrm{C}$ or $\mathrm{H}$ can usually be readily determined and a decision promptly made as to what should be done. From two to six hours of careful observation is usually necessary before the other classes of cases can be properly estimated.

Class A. These cases are of such a nature as to render any effort without avail. Operation is without value and is distinctly contraindicated. Death invariably results within a few hours, no matter what is done.

Class B. Here, an immediate operation is indicated. A straight incision such as is used in a typical subtemporal decompression is made on the involved side. The temporal muscle is separated in the direction of its fibers and retracted to either side. The skull is entered with perforator and burr, disclosing to view the large epidural clot. As soon as the diagnosis has been thus confirmed, and before enlarging the opening in the skull and removing the clots, the external carotid artery should be ligated through a small neck incision. By this precaution, much time will be saved and continued troublesome hemorrhage from the torn meningeal artery prevented. The intracranial ligation of the artery under such circumstances is not always an easy matter. After the external carotid artery has been ligated, the craniotomy can be safely continued. The bone is removed for a diameter of from 5 to $7 \mathrm{~cm}$., and the large epidural clot thoroughly removed. As a further precaution against the possible occurrence of increased intracranial pressure, the dura should be opened by means of crucial incisions. The overlying structures are now carefully closed with several layers of interrupted fine silk sutures.

Class $C$. In these cases the best results are obtained, if the technic of débridement (with certain modifications) advised by Cushing in the operative handling of war injuries is employed. The edges of the lacerated scalp are cut away; the underlying bone injury is exposed; all bone fragments are carefully removed and placed in sterile salt solution, and, by means of cathetersuction, all contused brain and blood clots are carefully removed. The dural opening is now closed, if possible, and the bone defect covered with such of the removed bone fragments as are suitable. The scalp wound is now carefully approximated with iriterrupted fine silk galea and skin sutures. If it seems advisable to give the brain room on account of increased intracranial pressure, a typical subtemporal decompression can now be done. As a rule, this will not be necessary.

Class D. These cases usually have more or less laceration of the temporal lobe, with accumulation of blood intradurally. The choice of treatment is still a source of much argument among neurologic surgeons. I myself am of the opinion that the best chance of recovery for these patients is offered by a subtemporal decompression, with or without temporary drainage by means of a small wick of rubber tissue placed under the temporal lobe and escaping through the lower angle of the wound. Occasionally, bilateral decompressions may be indicated. There is, of course, much room for argu. ment for and against operation in this class of cases. I have adopted the operative treatment in this particular type of injury during the last ten years and I feel convinced that the results have justified the method.

Class E. It is to this class of patients that I wish to call particular attention. The patients are usually brought into the hospital in a semiconscious condition. The blood pressure as a rule is somewhat higher than normal, but does not gradually increase as in Class $D$. The roentgen ray ustually reveals linear fractures of varying degrees, and the spinal fluid is often blood tinged. One feels safe in assuming that there has been definite gross brain damage, although there is no rapidly increasing intracranial pressure. Such cases under the usual method of treatment (absolute rest in bed and application of an ice cap to the head) are liable, within a few days, to show definite signs of increased intracranial pressure, not infrequently of such an alarming character as to call for energetic treatment. If these patients are operated on when symptoms of increased intracranial pressure develop, there is usually found a large collection of blood-tinged fluid under the dura, but apparently none in the subarachnoid space. Why this fluid should be between the arachnoid and dura is an interesting question. A plausible explanation is that, at the time of injury, the arachnoid is ruptured in different areas so that the cerebrospinal fluid does not remain in the subarachnoid space, from which it would probably be absorbed, but escapes directly under the dura, where cerebrospinal fluid absorption is impossible. When this fluid is allowed to escape at operation, the brain mass, instead of being swollen and edematous, seems at times to be actually decreased in volume. Drainage, for several days, usually relieves the distressing symptoms of increased intracranial pressure.

In our experience, the foregoing sequel to the injury can be prevented, provided the patient is given the proper treatment soon after being admitted to the hospital. Weed and McKibben ${ }^{1}$ have shown experimentally that the pressure of the cerebrospinal fluid and the bulk of the brain can be reduced by the intravenous injection of hypertonic salt solution. Foley and Putnam $^{2}$ demonstrated experimentally that hypertonic saline solution given by mouth likewise reduces cerebrospinal fluid and brain bulk, although the action is much slower than when saturated sodium chlorid solution is given intravenously. The clinical uses of salt solution in conditions of increased intracranial tension have likewise been discussed by Foley. ${ }^{3}$

In an effort to prevent the accumulation of fluid, with the resulting symptoms of increased intracranial pressure in this class of cases, we now administer a saturated solution of magnesium sulphate by mouth as a routine measure in all cases in which immediate operation is apparently not indicated. One-half ounce of the solution is given every two hours (the dose being decreased in proportion in children) for the first twenty-four to forty-eight hours. Thereafter, the time of administration is lengthened to every three hours for a day or two, and then gradually decreased accord-

1. Weed, L. H., and McKibbon, P. S.: Am. J. Physiol. 48:512 (May) 1919.

2. Foley, F. E. B., and Putnam, T. J.: Am. J. Physiol. 53: 464

3. Foley, F. E. B.: Clinical Use of Salt Solution in Conditions of Increased Intracranial Tension, Surg., Gynec. \& Obst. 33:126 (Aug.)
1921. 
ing to the condition of the patient. After from seven to ten days, the drug is usually withdrawn entirely. If at any time evidences of increased pressure develop in spite of the magnesium sulphate, the treatment is supplemented by the intravenous injection of 30 per cent. sodium chlorid solution. This, however, has been necessary in only a very few cases falling in this group. Although occasionally magnesium sulphate poisoning occurs, in our series of cases there has been no illustration of such an effect of the drug. However, on account of this danger, we have considered putting a series of patients on sodium chlorid tablets coated with phenyl salicylate in order to determine whether or not the same beneficial results are obtained.

As evidence of the value of this routine measure, in no case in this series has operative drainage of the fluid become necessary. All cases ran an uneventful course and the patients left the hospital apparently well within three weeks after admission. On the other hand, in several cases seen in consultation and in which no hypertonic solution had been given, the classic picture of increased intracranial pressure was present, necessitating energetic treatment in order to combat the distressing symptoms. As an illustration of this type of case, I wish to mention briefly a recent experience:

V. S., a man, received a head injury, Sept. 12, 1922, resulting in unconsciousness which lasted thirty-six hours. His condition then became satisfactory, according to the attending physician, and remained so until September 20 , when he began to show symptoms of increased intracranial pressure. September 22, he was admitted to the hospital in the general surgical service. $\mathrm{He}$ was kept in bed with ice cap to the head, but had no other treatment. September 29, I saw him in consultation. The picture presented at this time was practically that of the terminal stage of increased intracranial pressure. There was marked bilateral choking of the disks, stertorous, irregular breathing, rapid pulse, absence of knee refiexes and relaxation of sphincters. In general, the picture was such that one hesitated to advise operative treatment. Fifty cubic centimeters of a 30 per cent. sodium chlorid solution was given intravenously, with an appreciable improvement in the condition. Thirty cubic centimeters of the hypertonic solution was administered intravenously six hours later, and continued every six hours during the following thirty-six hours. At the end of this time, the picture presented was quite different in every respect. The patient was conscious; the pulse was 100 ; respiration was even and regular, and voluntary swallowing was possible. A saturated solution of magnesium sulphate in one-half ounce doses was then ordered by mouth every three hours. Within five days, the patient seemed securely on the road to recovery. Ten days after administration of the hypertonic solution had been instituted, the patient was normal in all respects, except the choked disks, which gave evidence of subsiding. October 20 , the patient left the hospital, apparently normal in every way.

Class F. To this class of cases belong those who have a history of a slight blow on the head followed by temporary unconsciousness. After a few hours, these patients become mentally clear, and careful neurologic examination fails to reveal any evidence of gross brain damage. The spinal fluid is clear, and there is no particular alteration of the pulse, blood pressure, or respiration. Such cases are ustally kept under careful observation for about three days, in order to be sure of the diagnosis. Rest in bed and free purgation with salines is the treatment indicated. If there is such a thing as real concussion of the brain, I feel that this particular class of cases should be the type referred to when such a diagnosis is made.
Class G. Not infrequently, patients with simple depressed fracture of the skull presenting no symptoms whatsoever, are admitted to the hospital. It is an open question whether or not these patients should be operated on. I feel that, as it is a simple matter to elevate such fractures and to investigate the structures underlying the depression, the safest policy is to operate in these cases as a matter of routine. Not infrequently, there will be found a certain amount of localized brain contusion underlying these fractures. When such a condition exists, a simple débridement by means of suction, followed by closure of the dura and replacement of the bone fragments, I believe to be the treatment of choice.

Class $H$. In treating the average case of scalp laceration, it is extremely important to determine whether or not the underlying structures are damaged. If not, the edges of the lacerated wound should be trimmed away and the wound closed with interrupted fine silk sutures. These wounds when so treated invariably heal by primary intention.

The value of hypertonic solutions in surgery of the brain has been proved by many observers (Haden, ${ }^{4}$ Cushing, ${ }^{5}$ Sachs ${ }^{6}$ ). It is not my purpose to discuss the various conditions in which they are beneficial. In my work I give saturated magnesium sulphate solution in varying doses, as a routine measure, after all cranial operations in which I feel that acute increased intracranial pressure may occur owing to fluid accumulation. When this condition already exists, I prefer the more rapid action of saturated sodium chlorid solution, intravenously.

\section{CONCLUSIONS}

1. All head injuries should be carefully studied and the type of injury properly estimated before the institution of any particular treatment.

2. No treatment is of avail in those cases of massive brain injury presenting evidence of rapid exhaustion of the medullary centers.

3. The treatment of middle meningeal hemorrhage can be greatly facilitated by ligating the external carotid artery.

4. The technic advised by Cushing in the treatment of war wounds of the skull and brain should be used in the treatment of those cases of brain injury in which there is localized brain contusion.

5. Subtemporal decompression is still the treatment of choice in cases of brain injury when there is evidence of gross brain laceration, and the patients are yet well within the so-called period of medullary compensation.

6. Saturated magnesium sulphate solution by mouth will prevent the occurrence of increased intracranial pressure due to fluid accumulation and thereby prevent the necessity of late operation in many cases of brain injury in which immediate operative intervention does not seem to be indicated.

7. Saturated solution of sodium chlorid intravenously is of inestimable value in relieving acute increased intracranial pressure due to fluid accumulation occurring several days after head injuries, with definite brain injury.

78 Forrest Avenue.

4. Haden, R. L.: Therapeutic Application of Alteration of Brain Volume by Injection of Glucose, J. A. M. A. 73: 983 (Sept. 27) 1919. 5. Cushing, Harvey, and Foley, F. E. B.: Proc. Soc. Exper. Biol. \& Med. 17:217, 1920 .

6. Sachs, Ernest, and Belcher, G. W.: Use of Saturated Salt Solution Intravenously During Intracranial Operations: Preliminary Report, J. A. M. A. 75:667 (Sept. 4) 1920 . 